\title{
A Wideband Circularly Polarized Slot Antenna Backed by a Frequency Selective Surface
}

\author{
Puneeth Kumar Tharehalli Rajanna* · Karthik Rudramuni · Krishnamoorthy Kandasamy
}

\begin{abstract}
This paper presents the design of a coplanar waveguide (CPW)-fed single-layer wideband circularly polarized (CP) planar slot antenna backed by a frequency selective surface (FSS). The planar slot antenna is fed with a stub-loaded modified CPW feed line to tune and optimize the impedance bandwidth. The corners of the square slot antenna are perturbed to produce two orthogonal degenerate modes required for a wideband CP operation. The FSS layer is placed under the slot antenna to increase the gain and axial ratio bandwidth. The measured results of the proposed antenna provide an impedance bandwidth of $63.22 \%$ and an axial ratio bandwidth of $31.14 \%$ with a peak gain of $4.87 \mathrm{~dB}$. The proposed antenna has a simple geometry, a wide $\mathrm{CP}$ bandwidth, and a good gain.
\end{abstract}

Key Words: Circular Polarization, Frequency Selective Surface, Slot Antenna.

\section{INTRODUCTION}

Circularly polarized $(\mathrm{CP})$ antennas are popular these days because of their superior performance over linearly polarized antennas. Single-feed CP antennas are much preferred in wireless communication because of their easy fabrication and low cost. Many techniques have been used to convert linearly polarized antennas to exhibit circular polarization behavior [1-5]. By introducing strip lines into coplanar waveguide (CPW)-fed slot antennas, a broadband CP antenna is achieved in [1-3]. In [4], an inverted L-shaped asymmetric CPW-fed square slot antenna with small strips is presented to achieve a wideband CP. A strip-loaded monopole antenna is designed to obtain a wideband $\mathrm{CP}$ in [5]. As strip loading is sensitive to the design process, achieving optimum wideband $\mathrm{CP}$ characteristics using a stub or the strip-loading method is difficult. The gain of a slot antenna is low because its radiation characteristic is bidirectional.
A perfect reflector or a backed cavity can be used to improve the gain, but both have a limited axial ratio bandwidth [6-8]. Therefore, metasurfaces (MTSs) are the potential structures that can be used to achieve a wide $\mathrm{CP}$ bandwidth and gain.

MTSs are periodic subwavelength metal or dielectric patches used to improve the performance of primary source antennas such as a patch or a slot. MTSs are used as reflectors to achieve a wide $\mathrm{CP}$ bandwidth. Artificial magnetic conductors (AMCs), high impedance surfaces, frequency selective surfaces (FSS), and electromagnetic band gap (EBG) structures are some of the MTSs used as reflectors to improve the gain, CP bandwidth, and pattern purity. A simple linearly polarized dipole antenna is utilized to generate a circularly polarized wave using an EBG structure in $[9,10]$. Different slot antennas with a single- and dual-layer AMCs as reflectors are investigated in $[11,12]$. A loop antenna is designed with an $\mathrm{AMC}$ as a reflector to improve the axial ratio bandwidth in [13]. These MTS-based antennas have a low axial ratio bandwidth. An AMC or an EBG struc-

Manuscript received December 05, 2018 ; Revised February 07, 2019 ; Accepted May 07, 2019. (ID No. 20181205-084)

Department of Electronics and Communication, National Institute of Technology Karnataka, Mangalore, India.

"Corresponding Author: Puneeth Kumar Tharehalli Rajanna (e-mail: puneeth.tc@gmail.com)

This is an Open-Access article distributed under the terms of the Creative Commons Attribution Non-Commercial License (http://creativecommons.org/licenses/by-nc/4.0) which permits unrestricted non-commercial use, distribution, and reproduction in any medium, provided the original work is properly cited.

(c) Copyright The Korean Institute of Electromagnetic Engineering and Science. All Rights Reserved. 
ture along with a slot antenna improves the gain and axial ratio bandwidth. The careful design of a primary source antenna with MTS as a reflector can achieve wideband CP characteristics. Corner truncated microstrip patch antennas have been discussed in the literature, but they have a narrow CP bandwidth [14]. A truncated slot antenna loaded with a pair of split-ring resonators (SRRs) is presented in [15] for dual-band CP applications. The disadvantage is that the corner truncated slot antenna alone cannot achieve a CP. The loading of an SRR on the truncated slot antenna gives a $\mathrm{CP}$ in both bands. In our proposed design, the perturbed slot antenna alone can produce a wideband $\mathrm{CP}$ by increasing the depth of perturbation.

In this paper, a novel perturbed slot antenna is proposed to achieve a wideband CP. Primarily, the square slot antenna is designed to radiate at $2.5 \mathrm{GHz}$ but with a linear polarization. The corner of the slot antenna is truncated to provide two orthogonal degenerate modes to produce a wide $\mathrm{CP}$ bandwidth. The truncated slot antenna achieves an axial ratio bandwidth of $20.68 \%$ at $4.35 \mathrm{GHz}$ resonance, but the gain of the perturbed slot antenna is $1.88 \mathrm{~dB}$. Furthermore, an effort is made to increase the axial ratio bandwidth and gain of the truncated planar slot antenna by backing the FSS. A $6 \times 10$ array of the FSS is used to improve the gain and axial ratio bandwidth of the perturbed slot antenna. The FSS improve the axial ratio bandwidth up to $31.14 \%$ at $4.33 \mathrm{GHz}$ resonance and a peak gain of up to $4.87 \mathrm{~dB}$.

\section{ANTENNA GEOMETRY AND ITS OPERATION}

The proposed antenna consists of a single-layer CPW linefed truncated corner slot antenna and an FSS layer, as shown in Fig. 1. A low-cost FR4 substrate with $\varepsilon_{r}=4.3$ and loss tangent

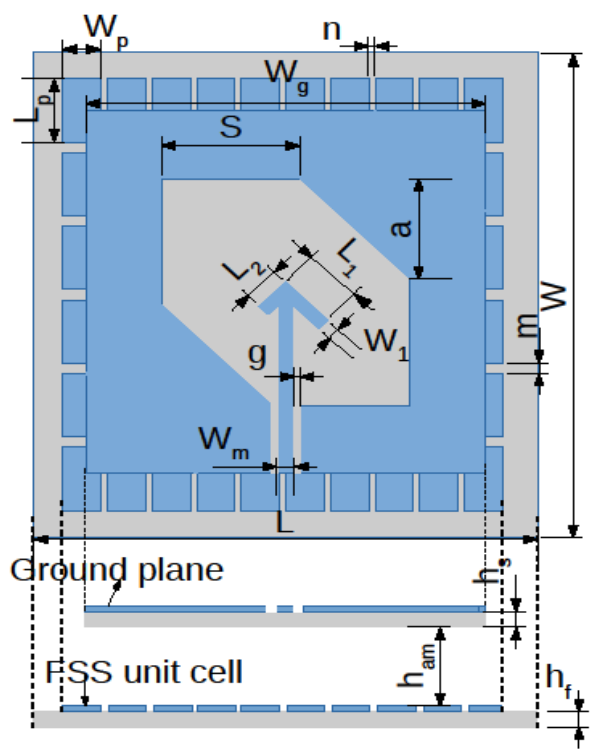

Fig. 1. Geometry of the proposed FSS-based antenna.

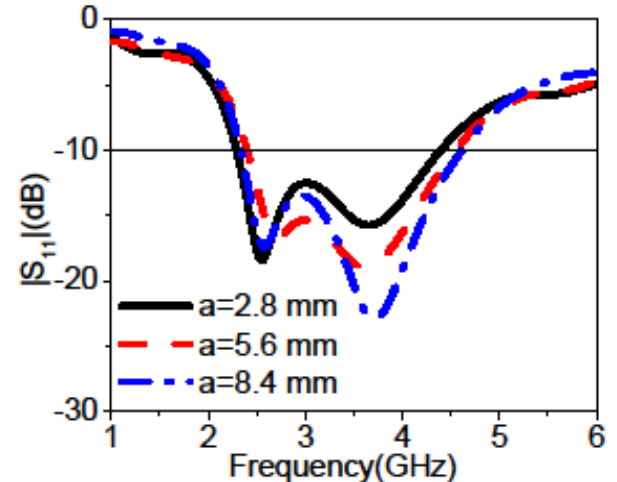

(a)

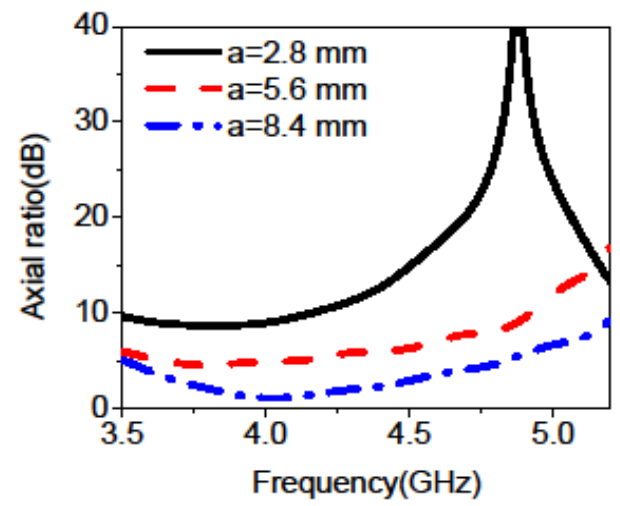

(b)

Fig. 2. Simulated reflection coefficient and axial ratio of the perturbed slot antenna: (a) $\left|S_{11}\right|$ and (b) axial ratio.

$\tan \delta=0.025$ is utilized for both slot antenna and the FSS. The FSS backs the slot antenna with an air gap of $h_{a m}=22 \mathrm{~mm}$. The resonance frequency of the slot antenna is calculated using Eq. (1) $[15]$.

$$
f_{r}=\frac{c}{2 S_{l}} \sqrt{\frac{2}{1+\varepsilon_{r}}}
$$

where $c$ is the speed of light, and $S_{l}=(S+a)$ is the total length of the square slot antenna. The proposed antenna parameters are $W_{p}=7 \mathrm{~mm}, L_{p}=13 \mathrm{~mm}, W_{g}=70 \mathrm{~mm}, W_{m}=3 \mathrm{~mm}, L_{m}=$ $29 \mathrm{~mm}, n=0.1 \mathrm{~mm}, m=0.2 \mathrm{~mm}, W=89 \mathrm{~mm}, L=100 \mathrm{~mm}$, $h_{s}=1.6 \mathrm{~mm}, h_{f}=0.508 \mathrm{~mm}, a=8.4 \mathrm{~mm}, L_{1}=7 \mathrm{~mm}, L_{2}=5$ $\mathrm{mm}$, and $W_{1}=1 \mathrm{~mm}$.

The conventional slot is perturbed to obtain the optimized performance of the axial ratio. The simulated reflection coefficient and axial ratio of the slot antenna with different truncated values are shown in Fig. 2. The dimension of truncation is optimized to $a=8.4 \mathrm{~mm}$, as the axial ratio value deteriorates with the further increase in truncation. The electric field distribution of the truncated slot antenna at different time intervals is shown in Fig. 3. In the figure, the truncated slot antenna radiates lefthand circularly polarized (LHCP) waves. The opposite corners of another diagonal of the slot antenna should be truncated to obtain right-hand circularly polarized (RHCP) waves. To fur- 


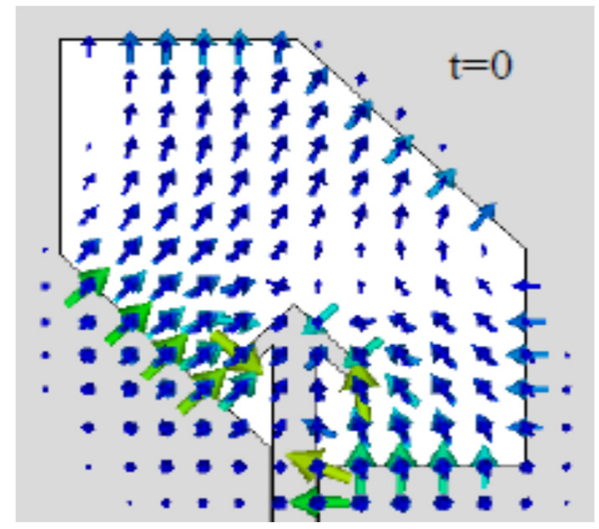

(a)

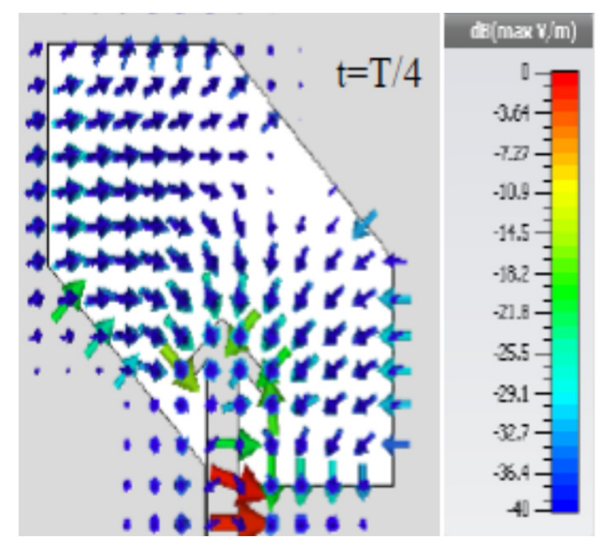

(b)

Fig. 3. Simulated electric field distribution of the truncated slot antenna at $3.47 \mathrm{GHz}$ : (a) $t=0$ and (b) $t=\mathrm{T} / 4$.

ther improve the gain and axial ratio bandwidth of the truncated slot antenna, an FSS consisting of rectangular unit cells are used.

The proposed FSS acts as a polarization-dependent reflective surface. To enhance the gain of the slot, the FSS is chosen to reflect the signal from the slot antenna back to the forward direction to improve the directivity of the antenna. The maximum gain is obtained for the $6 \times 10$ array. The FSS has a size of $W$ $\times L$ and a rectangular cell size of $W_{p}$ and $L_{p}$. The gap between patches in the horizontal and vertical directions is $m$ and $n$, respectively.

The perturbed slot gives a CP bandwidth of 3.9-4.8 GHz. The polarization-dependent reflective property of the FSS is used to improve the axial ratio bandwidth. The reflection phase curves for the $\mathrm{x}$ - and $\mathrm{y}$-polarized normally incident waves are shown in Fig. 4. The proposed FSS gives a reflection phase of $\pm 90^{\circ}$ for the $\mathrm{x}$ - and $\mathrm{y}$-polarized waves from $3.6 \mathrm{GHz}$ to $5 \mathrm{GHz}$. As explained in [9], this $90^{\circ}$ phase difference produces a $\mathrm{CP}$ radiation in the forward direction. This phase difference enhances the $\mathrm{CP}$ bandwidth of $3.6-5 \mathrm{GHz}$ of the perturbed slot antenna. The total field $\mathrm{E}$ is the sum of the direct radiating field $\left(E_{f}\right)$ from the slot and the reflected field $\left(E_{r}\right)$ from FSS. It is given by

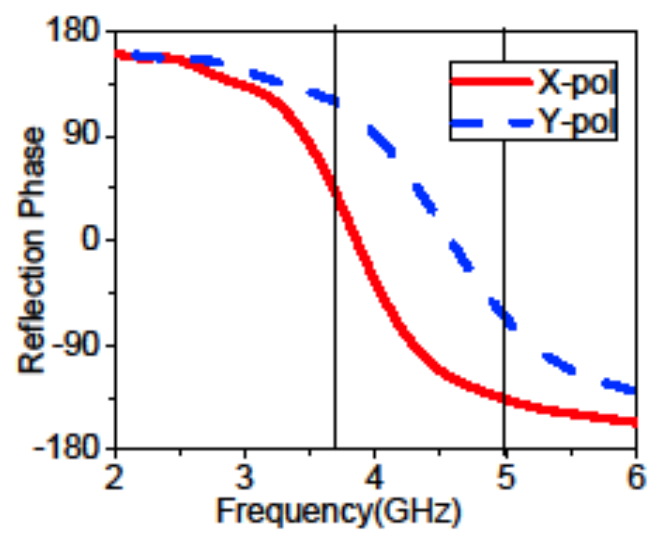

Fig. 4. Reflection phase characteristics of the FSS unit cell.

$$
\vec{E}=\overrightarrow{E_{f}}+\overrightarrow{E_{r}}=\frac{E_{0}}{\sqrt{2}} e^{-j k z}[(\hat{x}+\hat{y})+j(\hat{x}-\hat{y})]
$$

where $\overrightarrow{E_{f}}=\frac{E_{0}}{\sqrt{2}}\left(\hat{x} \cdot e^{-j k z}+\hat{y} \cdot e^{-j k z}\right)$ and

$$
\vec{E}_{r}=\frac{E_{0}}{\sqrt{2}}\left(\hat{x} \cdot e^{-j k z} \cdot e^{j \theta x}+\hat{y} \cdot e^{-j k z} \cdot e^{j \theta y}\right),
$$

where $\theta_{x}$ and $\theta_{y}$ are the polarization-dependent reflection phases for the $\mathrm{x}$ - and $\mathrm{y}$-polarized waves, respectively.

The design process of the proposed antenna is shown in Fig. 5. The CPW-fed conventional slot antenna (case a) resonates at $2.5 \mathrm{GHz}$ with linearly polarized waves. The axial ratio plot in Fig. 5(b) shows that the conventional slot has a high value of axial ratio at its resonance frequency. The corner perturbed slot

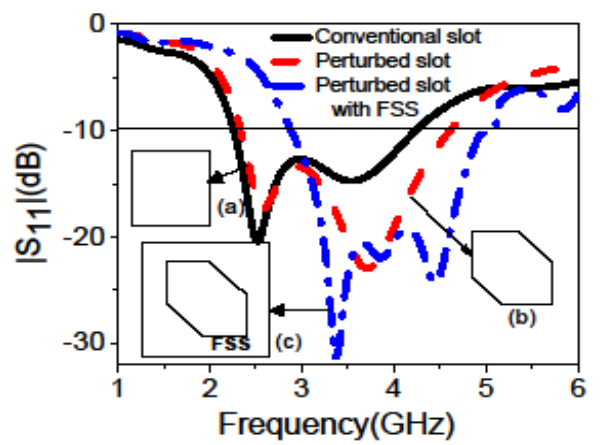

(a)

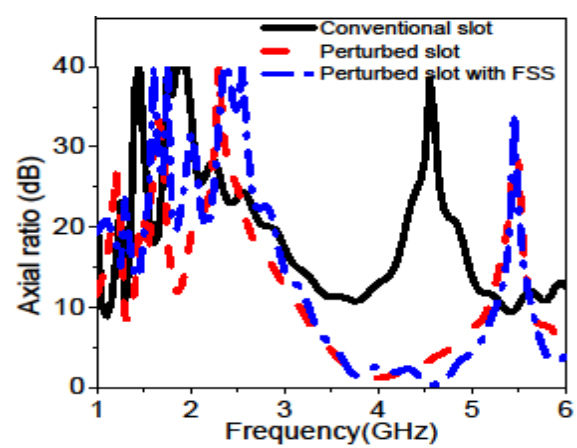

(b)

Fig. 5. Simulated reflection coefficient and axial ratio of the antenna design process: (a) $\left|S_{11}\right|$ and (b) axial ratio. 


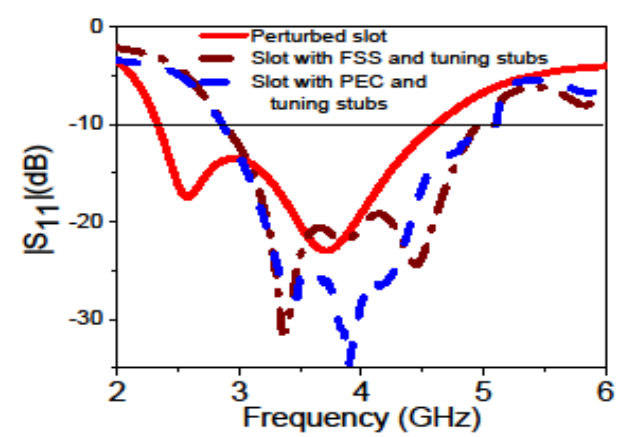

(a)

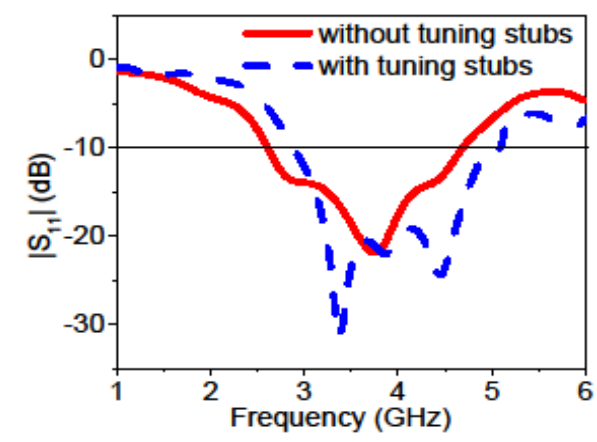

(c)

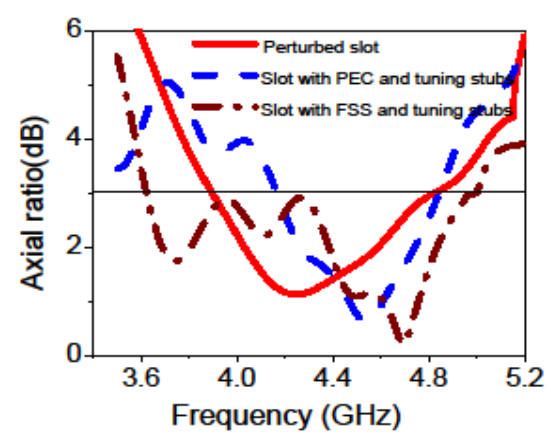

(b)

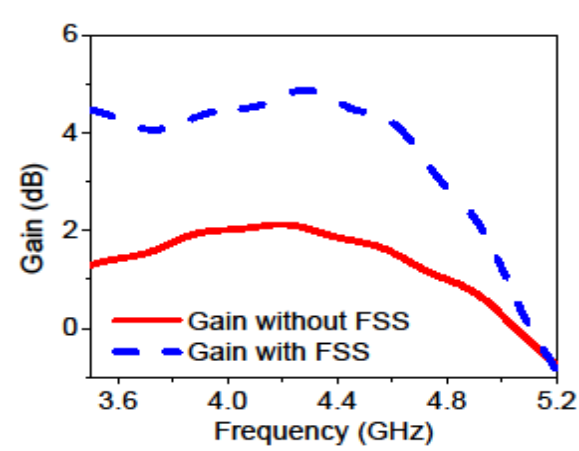

(d)

Fig. 6. Simulated reflection coefficient, axial ratio, and gain plot of the slot, the slot with PEC, and the slot with FSS: (a) $\left|S_{11}\right|$, (b) axial ratio, (c) $\left|S_{11}\right|$ of the proposed antenna with and without tuning stubs, and (d) gain of the proposed antenna with and without FSS.

(case b) resonates at $3.66 \mathrm{GHz}$ and produces a wideband circular polarization. To improve the axial ratio further, the FSS is placed below the slot antenna (case c), which enhances the axial ratio bandwidth.

The comparison among the conventional slot antenna, the slot antenna over perfect electric conductor (PEC), and the FSS-backed slot antenna provides the performance evaluation of FSS is shown in Fig. 6(a) and (b). The $S_{11}$ plot in Fig. 6(a) shows that the resonance of the slot over PEC and the slot over FSS is shifted compared with the perturbed slot because of the loading of PEC or FSS. In Fig. 6(b), the perturbed slot antenna has a lesser axial ratio bandwidth than the FSS-backed slot antenna because of the forward signal from the slot, and the reflected signal from the FSS produces orthogonal fields in the far-field region producing a wideband $\mathrm{CP}$.

The reflection coefficient of the designed antenna with and without tuning stubs is shown in Fig. 6(c). The role of the tuning stubs in the CPW feed is to tune and optimize the impedance bandwidth, so that the $\mathrm{CP}$ bandwidth lies within the specified impedance bandwidth. The slot antenna directivity is improved by nearly $3 \mathrm{~dB}$ for both PEC and FSS, but improving the axial ratio bandwidth is not possible for PEC. The gain comparison of the perturbed slot and the perturbed slot with FSS is illustrated in Fig. 6(d). In this figure, the gain of the perturbed slot is less which is $1.88 \mathrm{~dB}$ at $4.2 \mathrm{GHz}$ because of the bidirectional radiation pattern. The FSS is placed below the perturbed slot antenna, which increases the gain up to $4.87 \mathrm{~dB}$. The above comparison infers that the FSS-backed perturbed slot antenna improves the axial ratio bandwidth and gain.

\section{EXPERIMENTAL RESULTS AND DISCUSSION}

The FSS-backed slot antenna is fabricated, and the simulation results are exper imentally verified. Fig. 7 presents a photograph of the fabricated prototype. The simulated and measured reflection coefficient magnitudes are plotted in Fig. 8(a). A simulated impedance bandwidth of $55.23 \%$ is achieved from 2.87 $\mathrm{GHz}$ to $5.06 \mathrm{GHz}$. The measured impedance bandwidth of $63.22 \%$ is obtained from $2.65 \mathrm{GHz}$ to $5.10 \mathrm{GHz}$. A simulated $3 \mathrm{~dB}$ axial ratio bandwidth of $30.94 \%$ is achieved from 3.66 $\mathrm{GHz}$ to $5 \mathrm{GHz}$, and the measured axial ratio bandwidth of

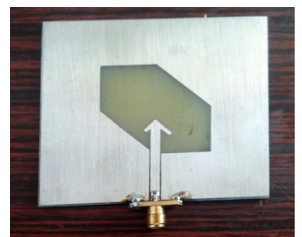

(a)

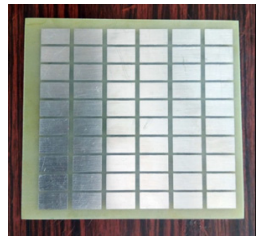

(b)

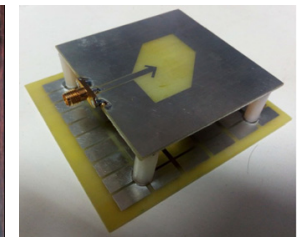

(c)
Fig. 7. Prototype of the fabricated antenna: (a) slot antenna, (b) FSS, and (c) proposed antenna. 


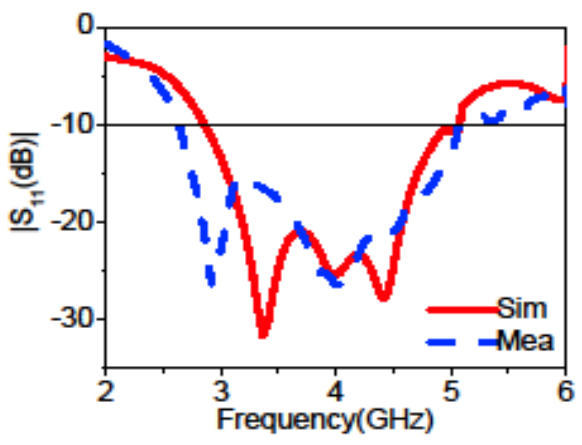

(a)

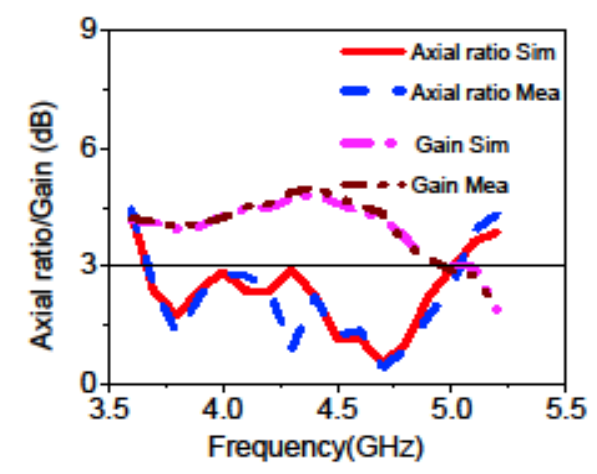

(b)

Fig. 8. Simulated and measured reflection coefficient, axial ratio, and gain of the proposed antenna: (a) $\left|S_{11}\right|$ and (b) axial ratio and gain.

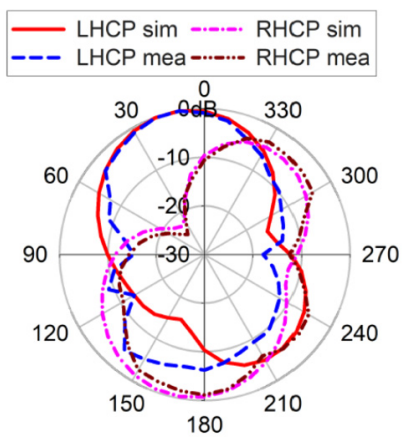

(a)

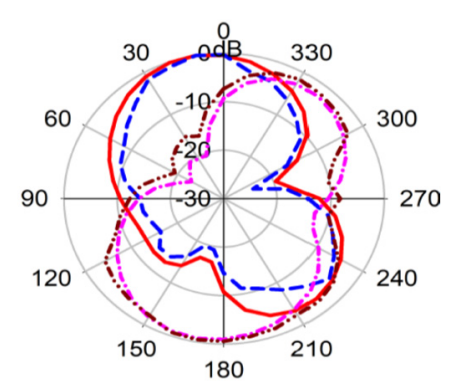

(c)

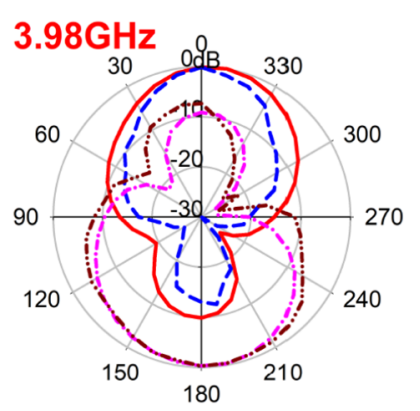

(b)

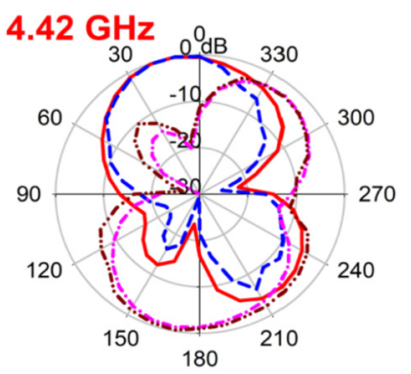

(d)
Fig. 9. Simulated and measured LHCP and RHCP radiation patterns in (a) the $\mathrm{xz}$ plane at $3.98 \mathrm{GHz}$, (b) yz plane at 3.98 $\mathrm{GHz}$, (c) xz plane at $4.42 \mathrm{GHz}$, and (d) yz plane at 4.42 $\mathrm{GHz}$.

$31.14 \%$ is obtained from $3.66 \mathrm{GHz}$ to $5.01 \mathrm{GHz}$. The simulat- ed and measured broadside gain and axial ratio plots are shown in Fig. 8(b). The broadside peak gain of $4.87 \mathrm{~dB}$ is obtained at $4.2 \mathrm{GHz}$ with a $2 \mathrm{~dB}$ variation over the $3 \mathrm{~dB}$ axial ratio bandwidth. The simulated and measured radiation patterns are given at different frequencies in both the $\mathrm{xz}$ and $\mathrm{yz}$ planes in Fig. 9. The simulated and measured radiation patterns agree well for both the RHCP and the LHCP. The LHCP level is $16 \mathrm{~dB}$ below the RHCP level in both the $\mathrm{xz}$ and $\mathrm{yz}$ planes. The LHCP is obtained at an inclined angle of $20^{\circ}$ with respect to the broadside direction. The tilted LHCP radiation is due to the perturbation effect of the slot antenna.

\section{CONCLUSION}

A wideband CP slot antenna with FSS loading is proposed in this study. The truncated slot antenna achieves an optimized axial ratio but with a low gain. To improve the gain and the axial ratio of the truncated slot antenna, an FSS is used as a reflector. The proposed antenna achieves a measured impedance bandwidth of $2.44 \mathrm{GHz}$ and a $3 \mathrm{~dB}$ axial ratio bandwidth of 1.35 $\mathrm{GHz}$ with a peak gain of $4.87 \mathrm{~dB}$. The proposed antenna is useful for CP applications, such as radar and satellite, over the $\mathrm{CP}$ frequency range of $3.66-5.01 \mathrm{GHz}$.

This work was supported by the Indian Space Research Organization under the RESPOND program.

\section{REFERENCES}

[1] R. Cao and S. C. Yu, "Wideband compact CPW-fed circularly polarized antenna for universal UHF RFID reader," IEEE Transactions on Antennas and Propagation, vol. 63, no. 9, pp. 4148-4151, 2015.

[2] S. Karamzadeh, V. Rafii, M. Kartal, and H. Saygin, "Compact UWB CP square slot antenna with two corners connected by a strip line," Electronics Letters, vol. 52, no. 1, pp. 10-12,2016.

[3] J. Pourahmadazar and S. Mohammadi, "Compact circularlypolarised slot antenna for UWB applications," Electronics Letters, vol. 47, no. 15, pp. 837-838, 2011.

[4] J. Y. Sze, J. C. Wang, and C. C. Chang, "Axial-ratio bandwidth enhancement of asymmetric-CPW-fed circularlypolarised square slot antenna," Electronics Letters, vol. 44, no. 18, pp. 1048-1049, 2008.

[5] R. K. Saini, S. Dwari, and M. K. Mandal, "CPW-fed dualband dual-sense circularly polarized monopole antenna," IEEE Antennas and Wireless Propagation Letters, vol. 16, pp. 2497-2500, 2017.

[6] S. Fu, Q. Kong, S. Fang, and Z. Wang, "Broadband circularly 
polarized microstrip antenna with coplanar parasitic ring slot patch for L-band satellite system application," IEEE Antennas and Wireless Propagation Letters, vol. 13, pp. 943946, 2014.

[7] S. P. Pan, J. Y. Sze, and P. J. Tu, "Circularly polarized square slot antenna with a largely enhanced axial-ratio bandwidth," IEEE Antennas and Wireless Propagation Letters, vol. 11,pp. 969-972, 2012.

[8] R. Li, B. Pan, A. N. Traille, J. Papapolymerou, J. Laskar, and M. M. Tentzeris, "Development of a cavity-backed broadband circularly polarized slot/strip loop antenna with a simple feeding structure," IEEE Transactions on Antennas and Propagation, vol.56, no. 2, pp. 312-318, 2018.

[9] F. Yang and Y. Rahmat-Samii, "A low profile single dipole antenna radiating circularly polarized waves," IEEE Transactions on Antennas and Propagation, vol. 53, no. 9, pp. 30833086, 2015.

[10] H. Yi and S. W. Qu, "A novel dual-band circularly polarized antenna based on electromagnetic band-gap structure," IEEE Antennas and Wireless Propagation Letters, vol. 12, pp. 1149-1152, 2013.

Puneeth Kumar Tharehalli Rajanna

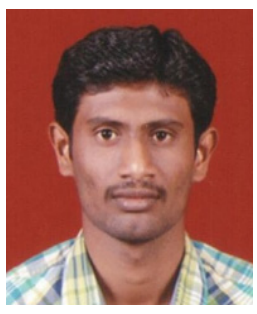

received his B.E. degree in telecommunication engineering and M.Tech. degree in digital communication engineering from Visveswaraya Technological University, Belgaum, Karnataka, India, in 2009 and 2013, respectively. He is currently working toward his $\mathrm{Ph} . \mathrm{D}$. in electronics and communication at the National Institute of Technology Karnataka, Mangalore, India. His field of research includes metamaterial-based antennas, microwave antennas, and microstrip antennas.

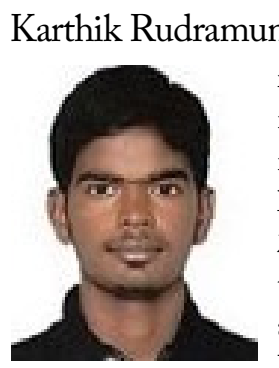

received his B.E. degree in electronics and communication engineering and M.Tech. degree in RF and microwave engineering from Visveswaraya Technological University, Belgaum, Karnataka, India, in 2009 and 2013, respectively. He is currently working toward his $\mathrm{Ph} . \mathrm{D}$. in electronics and communication at the National Institute of Technology Karnataka, Mangalore, India. His field of research includes metamaterial-based antennas, Goubau line-based leaky wave antennas, and microstrip antennas.
[11] K. Agarwal and A. Alphones, "Unidirectional wideband circularly polarised aperture antennas backed with artificial magnetic conductor reflectors," IET Microwaves, Antennas \& Propagation, vol. 7, no. 5, pp. 338-346, 2013.

[12] K. Agarwal and A. Alphones, "Wideband circularly polarized AMC reflector backed aperture antenna," IEEE Transactions on Antennas and Propagation, vol. 61, no. 3, pp. 1456-1461, 2013.

[13] H. Li, K. W. Xu, X. B. Wang, R. Kanan, and L. X. Ran, "Low-profile circularly polarised loop antenna assisted with an effective PMC bandwidth," Electronics Letters, vol. 49, no. 16, pp. 978-979, 2013.

[14] P. C. Sharma and K. C. Gupta, "Analysis and optimized design of single feed circularly polarized microstrip antennas," IEEE Transactions on Antennas and Propagation, vol. 31, no. 6, pp. 949-955, 1983.

[15] K. Kandasamy, B. Majumder, J. Mukherjee, and K. P. Ray, "Dual-band circularly polarized split ring resonators loaded square slot antenna," IEEE Transactions on Antennas and Propagation, vol. 64, no. 8, pp. 3640-3645, 2016.

Krishnamoorthy Kandasamy

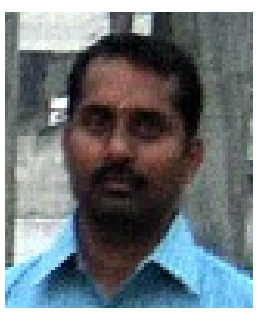

received his B.E. degree in electronics and communication engineering from Bharathiar University, Coimbatore, India, in 2003, his M.E. degree in communication systems from the College of Engineering, Guindy, Anna University, Chennai, India, in 2007, and his Ph.D. in electrical engineering from IIT Bombay, Mumbai, India, in 2016. He is currently an assistant professor at the Department of Electronics and Communication Engineering, National Institute of Technology Karnataka, Surathkal, India. His current research interests include metamaterials, antenna engineering, microwave integrated circuits (MICs), and monolithic MICs. 